Egyptian

Orthodontic Journal

\title{
EFFECT OF BONDING BRACKETS BY SULPHATED POLYACRYLIC VS. CONVENTIONAL ACID ETCHING ON DECIDUOUS AND PERMANENT ENAMEL
}

\author{
Shaza M. Hammad ${ }^{1}$, Abeer M. Abdellatif ${ }^{2}$
}

\section{ABSTRACT:}

The aim of this study was to compare the shear bond strengths of metallic brackets bonded to deciduous and permanent enamel treated with crystalline growth solution (40\% sulphated polyacrylic acid) and the adhesive remnants after debonding. Also, to verify the topographic difference of the treated deciduous and permanent enamel surface using Scanning Electron Microscope (SEM) compared with conventional acid-etching. Two sets of sixty freshly extracted deciduous and permanent teeth were collected. Each set was divided into 2 groups of 15 teeth each; groups $I$ and II (deciduous teeth) and groups III and IV (permanent teeth). For groups $I$ and III, 40\% sulphated polyacrylic acid was applied and for groups II and IV, conventional acid-etching was used. Brackets were bonded, and the shear bond strength was measured using a universal testing instrument. The residual adhesive was examined using a stereomicroscope at 20 times magnification. The SEM topographic pattern of treated enamel was evaluated. Shear bond strengths of the brackets of the four groups were compared by Two-Way Analysis of Variance (ANOVA). T-test was done for inter-groups comparison. The least recorded shear bond strength was $12.65 \pm 0.15 \mathrm{MPa}$ in group I; the deciduous teeth treated with sulphated polyacrylic solution. Most of enamel surfaces treated

1- Department of Orthodontics, Faculty of Dentistry, Mansoura University, Egypt.

2- Department of Pediatric Dentistry and Dental Public Health, Faculty of Dentistry, Mansoura University, Egypt. 
Egyptian

Orthodontic Journal

with the sulphated polyacrylic acid were left clean with no or minimal adhesive remnants, after brackets debonding. The deciduous enamel treated with the sulphated polyacrylic acid revealed formation of less abundant crystals than that appeared on permanent teeth enamel as shown by the SEM. Thus it is concluded that, 40\% sulphated polyacrylic acid can be used for bonding metallic brackets to deciduous and permanent teeth.

Key Words: Crystalline growth; deciduous teeth; Bonding; shear bond strength.

\section{INTRODUCTION}

Early orthodontic treatment for various types of malocclusion is recommended to enhance dental and skeletal development before completion of the permanent dentition ${ }^{[1]}$. A two-by-four sectional edgewise appliance has been used in the mixed dentition for early orthodontic treatment of dental anterior crossbites, maxillary incisor crowding, and maxillary impacted (unerupted) incisors ${ }^{[2-4]}$. To facilitate treatment of these malocclusions, orthodontic brackets might be bonded not only to premolars but also to deciduous canines and molars as well as the four permanent incisors and the two permanent maxillary first molars. Thus, premolars and deciduous teeth can be used as anchor teeth in early orthodontic treatment with sectional edgewise appliances ${ }^{[4]}$.

The enamel surface structure of the deciduous teeth is different from that of the permanent teeth. The outer prismless enamel layer is more common in deciduous teeth than in permanent teeth ${ }^{[5-7]}$. It lacks the characteristic prism markings of enamel and has no well-developed etch pattern with well-defined prisms, resulting in insufficient resin penetration and weak bond strength ${ }^{[8]}$. It has been reported that deciduous tooth enamel has higher percentages of moisture and organic contents and a lower percentage of inorganic contents than permanent tooth enamel ${ }^{[9]}$. The structure of deciduous tooth enamel, with its low inorganic and high 
organic contents, may produce poorly developed etching patterns and may be also responsible for the lower bond strength ${ }^{[9-11]}$.

Orthodontic bond strength must satisfy two-pronged requirements: it must be sufficient to retain the brackets but low enough to allow easy clean-up of adhesives when the brackets are removed. The bond strength of orthodontic brackets bonded to enamel surfaces with light-cured adhesives depends on several factors, including variations in tooth type, type of etchant and its concentration and application time ${ }^{[12,13]}$. It is documented that, acid-etching causes the dissolution of the interprismatic material in the enamel, producing an irregular enamel surface and it has been reported to cause up to $55 \mu \mathrm{m}$ of enamel loss ${ }^{[14]}$. The permanent loss of enamel calcium during the acid etching procedure may render the enamel surface more susceptible to demineralization during and after the orthodontic treatment ${ }^{[15]}$. This may also explain the iatrogenic effects on the enamel surface, especially during bracket removal. Reports of enamel fractures and cracks when debonding raised questions about the safety of using this strong adhesive technique ${ }^{[16,17]}$. There is an evident need to develop a mechanical or chemical retention system that would not or minimally alter the outer enamel surface. Building up a crystal layer for mechanical retention instead of creating a rough surface by means of strong etching has been studied ${ }^{[18,19]}$. The crystal bonding technique involved the application of polyacrylic acid containing residual sulphate ions which react with the enamel surface to produce a deposit of crystalline calcium sulphate growth ${ }^{[19]}$. This method was studied as an alternative to the conventional phosphoric acid etch technique creating a micromechanical retentive surface through the formation of a crystalline interface ${ }^{[19,20]}$.

Although crystalline growth effect had been studied on permanent teeth, no study has been conducted for deciduous teeth ${ }^{[18-22]}$. Our hypothesis is that crystalline growth technique can be used effectively for bonding brackets to deciduous as well as permanent teeth. The purposes of this study were to evaluate, in comparison to conventional acid etching, 
Egyptian

Orthodontic Journal

the shear bond strengths of orthodontic brackets bonded to deciduous and permanent teeth with a crystal bonding technique using the high concentration of potassium sulphate ion; (1M) in 40\% polyacrylic acid, the adhesive remnants after debonding and the effect of both etchants on the topography of deciduous and permanent enamel using Scanning Electron Microscopy (SEM).

\section{Methodology}

The protocol of the study was approved by the Local Ethical Committee of Mansoura University. The crystalline solution used for this study was $40 \%$ sulphated polyacrylic acid of molecular weight 5,000 . It composed of $1 \mathrm{M}$ potassium sulphate ions reacted with polyacrylic acid (3M Unitek ), prepared at the Department of Pharmaco-chemistry, Faculty of Pharmacy, Mansoura University-Egypt.

Thirty deciduous second molars, free of caries, freshly extracted due to prolonged retention and thirty freshly extracted first premolars for orthodontic reasons of children aged 12-14 years were collected from the pediatric dental and orthodontic clinics. The criteria for tooth selection included intact buccal enamel with no pre-treatment with chemical agents, no cracks, no hypoplasia and no caries. Teeth were washed with water and stored in a solution of $0.1 \%$ thymol at $4 \mathrm{C}^{\circ}$ for until their use within two months period. The used brackets were metal mesh base premolar edgewise with 0.018-inch slot (Victory series, 3M Unitek, Monrovia, Calif). The average bracket base area was $9.94 \mathrm{~mm}^{2}$ according to the manufacture's specification.

For shear bond strength test, the deciduous teeth were divided into 2 groups of 15 teeth each (groups I and II) of second molars in each group. The permanent teeth were also divided into 2 groups of 15 teeth each (groups III and IV) of first premolars in each group. For groups I and III, 40\% sulphated polyacrylic acid, $1 \mathrm{M}$ ion concentration was applied. As a control, conventional adhesive system (3M, Unitek $\left.{ }^{\mathrm{TM}}\right)$ was used for groups II and IV. 
For all groups, the buccal surface of each tooth crown was cleansed with a mixture of water and fluoride-free pumice by a rubber prophylactic cup for 10 seconds. Each tooth was then rinsed with a water spray for 10 seconds and dried with an oil- and moisture-free air stream. In groups I and III (crystalline bonding groups), the buccal enamel surface of each tooth crown was treated for 15 seconds with the $40 \%$ sulphated polyacrylic acid solution, rinsed and then thoroughly dried. For groups II and IV (conventional acid-etching group), the buccal enamel surface was etched with the acid etchant for 15 seconds, followed by thorough rinsing and drying with successful frosted appearance.

For all groups, a thin uniform layer of Transbond XT primer (3M Unitek) was then applied to the enamel surface, and Transbond XT adhesive (3M Unitek) was applied to the bracket base. Each bracket was placed on the buccal enamel surface of each tooth and pressed firmly to express any adhesive from the margins of the bracket base, and removed with an explorer before curing. Then, the composite was light-cured with an Ortholux LED curing unit (3M Unitek, 120 Volt LED Curing Light) for 10 seconds ( 5 seconds mesially, 5 seconds distally). All teeth were stored in distilled water at $37^{\circ} \mathrm{C}$ for one week. Each tooth was then embedded in a specimen holder ring with a self-curing acrylic resin. The buccal enamel surface was parallel to and projected above the rim of the cylindrical specimen holder ring.

\section{Assessment of shear bond strength}

A universal testing machine (Lloyd Instruments; West Fareham, UK) was used to determine the shear bond strengths. A load was applied to the occlusal brackets' wings with a force in the occluso-gingival direction parallel to the buccal enamel surface. The force required to shear off the bracket was recorded in Newton $(\mathrm{N})$ at a crosshead speed of $1.0 \mathrm{~mm}$ per minute. The shear bond strength in Megapascal (MPa), was then calculated by dividing the shear force by the bracket base area $\left(9.94 \mathrm{~mm}^{2}\right)$. 


\section{Assessment of the adhesive remnants on enamel surfaces}

After bracket debonding, the bracket bases and the enamel surfaces were examined with a Stereo-microscope (SMZ1500, Nikon, Tokyo, Japan) at 20 times magnification to determine the adhesive remnants on enamel of both deciduous and permanent teeth. Adhesive remnants index (ARI) scores were used to assess the amount of adhesive left on the enamel surface ${ }^{[23]}$.

\section{Enamel surface topographic examination (SEM)}

A total of 24 additional deciduous and permanent buccal enamel surfaces were prepared (6 surfaces each); the crowns were sectioned from the roots with a diamond disc at the labial cemento-enamel junction, and each crown was cut longitudinally in a mesio-distal direction. The buccal surfaces of the crowns were cleaned thoroughly with a rubber cup and a slurry of pumice and water, followed by rinsing with water spray and drying with compressed air. The application time of both the sulphated polyacrylic solution and $37 \%$ phosphoric acid was 15 seconds for the deciduous and the permanent teeth. Either of the etchants used, was generously spread over the prepared enamel surface with a mini-sponge, and agitated slightly during the application. After enamel conditioning or etching, the teeth were rinsed with water spray for 15 seconds and dried. Specimens were mounted on aluminium stubs using carbon tab and prepared for SEM by sputtering with gold in a high vacuum evaporator (SPI®Module- Vac/ sputter coater) for 45-60 seconds. They were examined in a Jeol-JSM-5200LV Scanning microscopy-Japan, under 500 times magnification and $25 \mathrm{Kv}$.

\section{Statistical analysis}

Mean shear bond strengths of the brackets of the four groups were compared by Two-way analysis of variance (ANOVA). T-test was done for inter-groups comparisons. The non-parametric Kruskal-Wallis test 
was used to test the significant differences between groups with regard to ARI scores. Statistical tests were conducted by using SPSS 15.0 statistical package (SPSS Inc., Chicago, Illinois, U.S.A). All tests were made at the $P \leq 0.05$ level of significance.

\section{RESULTS}

\section{Shear bond strength}

The least recorded shear bond strength was $12.65 \pm 0.15 \mathrm{MPa}$, demonstrated by the brackets bonded to deciduous teeth treated with the sulphated polyacrylic acid. The highest recorded value was $17.27 \pm 0.29$ $\mathrm{MPa}$, obtained by the brackets bonded to permanent teeth treated with the conventional acid-etching system. Almost equal shear bond strength values were demonstrated by the brackets bonded to permanent enamel following application of sulphated polyacrylic acid and the brackets bonded to deciduous enamel following conventional acid etching $(14.28 \pm 0.24,14.65 \pm 0.23 \mathrm{MPa})$. On using any of the two systems, a highly significant lower shear bond strength was demonstrated by brackets bonded to deciduous teeth compared to those bonded to permanent teeth $(P \leq 0.05)$. Also, when the two sets of teeth were treated by the two systems, it was noted that the shear bond strength of the brackets bonded to permanent teeth showed higher difference, compared with those bonded to the deciduous teeth. Also, the bond strength of brackets bonded to deciduous and permanent teeth treated by the conventional acid etchant showed higher difference, compared with those treated by the sulphated polyacrylic solution (Table 1).

\section{Adhesive remnant estimation}

The distribution of ARI scores for each group is shown in Table 2. The Kruskal-Wallis test showed significant differences in ARI scores between the crystalline bonding groups (I, III) and the conventional acid-etching groups (II, IV). ARI scores 0 and 1 occurred more frequently on deciduous and permanent enamel treated by the sulphated polyacrylic acid (Groups I and III). Thus, most of these debonded enamel surfaces were left clean with no or minimal adhesive remnants. 
Table 1: Shear bond strength (MPa) of brackets bonded on the enamel surfaces of the two groups of teeth, treated with two etchant systems.

\begin{tabular}{|l|c|c|c|c|}
\hline Etchant & $\begin{array}{c}\text { Sulphated polyacrylic solution } \\
\text { Mean } \pm \text { SD } \\
(\mathrm{n})=15\end{array}$ & $\begin{array}{c}\text { Conventional acid etchant } \\
\text { Mean } \pm \text { SD } \\
(\mathrm{n})=15\end{array}$ & $\begin{array}{c}t \\
\text { value }\end{array}$ & $\begin{array}{c}P \\
\text { value }\end{array}$ \\
\hline Deciduous & (G I) $12.65 \pm 0.15$ & $(\mathrm{G} \mathrm{II}) 14.65 \pm 0.23$ & 28.51 & 0.001 \\
\hline Permanent & (G III) $14.28 \pm 0.24$ & $(\mathrm{G} \mathrm{IV}) 17.27 \pm 0.29$ & 30.37 & 0.001 \\
\hline$t$ value & 27.53 & 27.60 & & \\
\hline$P$ value & 0.001 & 0.001 & & \\
\hline
\end{tabular}

Significance at $\leq 0.05$

Table 2: Adhesive Remnant Index (ARI) scores: groups I, III: deciduous and permanent teeth treated by sulphated polyacrylic solution and groups II, IV: deciduous and permanent teeth treated by conventional acid-etching.

\begin{tabular}{ccccc}
\hline ARI score & Group (I) & Group (II) & Group (III) & Group (IV) \\
\hline $\mathbf{0}$ & $11(73 \%)$ & $1(6.5 \%)$ & $9(60 \%)$ & $0(0.0 \%)$ \\
$\mathbf{1}$ & $4(27 \%)$ & $4(27 \%)$ & $4(27 \%)$ & $5(33 \%)$ \\
$\mathbf{3}$ & $0(0.0 \%)$ & $6(40 \%)$ & $2(13 \%)$ & $4(27 \%)$ \\
Total no. & $0(0.0 \%)$ & $4(26.5 \%)$ & $0(0.0 \%)$ & $6(40 \%)$ \\
\hline
\end{tabular}

Score 0 ; no adhesive remaining on the tooth;

Score 1; less than half of the adhesive remaining on the tooth;

Score 2; more than half of the adhesive remaining on the tooth; and

Score 3; all adhesive remaining on the tooth with a distinct impression of the bracket base.

\section{Enamel surface topographic examination (SEM)}

Using scanning electron microscope, the treated deciduous enamel with the sulphated polyacrylic acid revealed formation of crystals (Fig. 1). Similarly, the treated permanent enamel with the same solution showed formation of crystals on the enamel surface, but in a more 
abundant manner (Fig. 2). On the other hand, etching deciduous and permanent enamel with the conventional acid etchant showed typical patterns with preferential dissolution of the prism peripheries and cores (Fig. 3, 4).

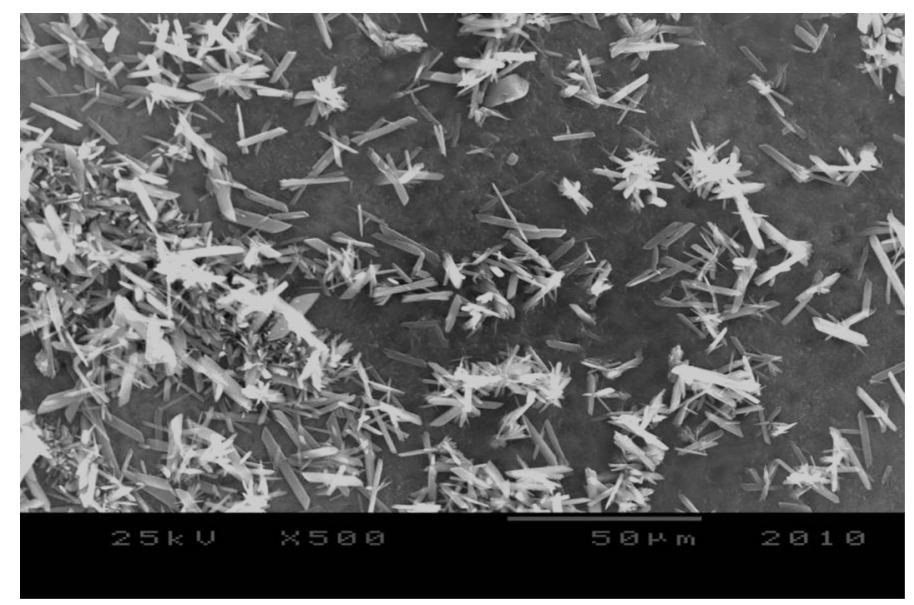

Fig. 1: SEM image shows crystal growth on deciduous tooth enamel treated with $40 \%$ sulphated polyacrylic acid

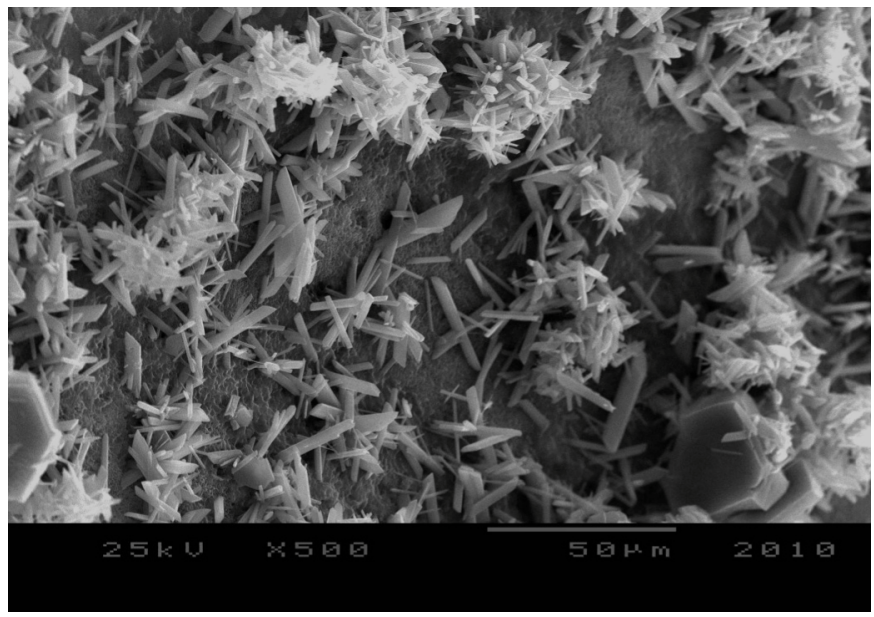

Fig. 2: SEM image shows crystal growth on permanent tooth enamel treated with $40 \%$ sulphated polyacrylic acid 
Egyptian

Orthodontic Journal

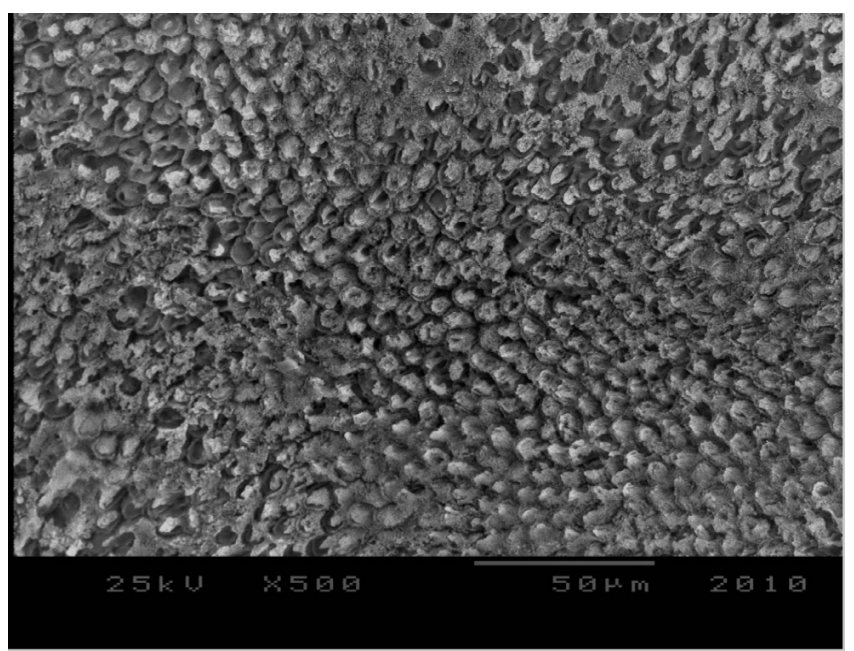

Fig. 3: SEM image shows the typical pattern of deciduous tooth enamel treated with conventional acid-etching

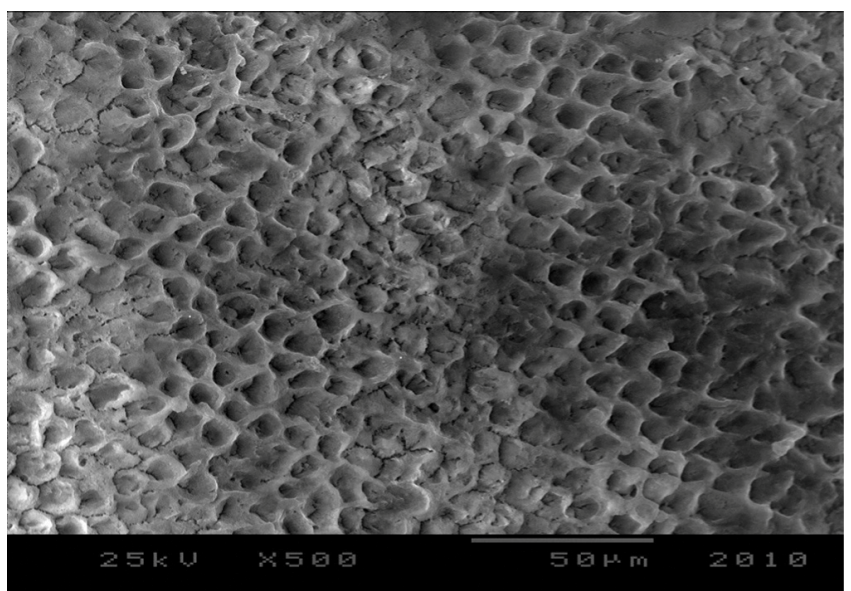

Fig. 4: SEM image shows the typical pattern of permanent tooth enamel treated with conventional acid-etching

\section{DISCUSSION}

This study is one of the pioneer studies (no studies investigated the effect on deciduous teeth before) to investigate differences in shear bond strength between deciduous and permanent teeth when identical orthodontic brackets were bonded with crystal growth technique and 
conventional phosphoric acid etching. The division of the sample was based on the suggestion by Hobson et $\mathrm{al}^{[13]}$, that comparisons of bond strength values should be made with the same tooth type or appropriately stratified groups of teeth.

Bond strengths between 6 and $8 \mathrm{MPa}$ are clinically sufficient for successfully bonding orthodontic brackets to enamel ${ }^{[24-28]}$. Our findings showed that the shear bond strengths of all the 4 groups were higher than the clinically required range of 6 to $8 \mathrm{MPa}$.

In the present study we found significant differences in the shear bond strengths of brackets bonded with phosphoric acid-etching between deciduous and permanent teeth after enamel treatment with both systems, which agrees with previous result ${ }^{[26]}$. The significantly lower shear bond strength in the deciduous teeth might be explained by the outer prismless enamel layer and the adhesive thickness between the bracket base and the enamel surface. The outer prismless enamel layer is more common in deciduous teeth than in permanent teeth ${ }^{[5,6]}$. The area on the labial surface, where the prismless enamel layer is most common, is the gingival third of the tooth crown in permanent teeth, whereas this area in deciduous teeth is the middle third, which corresponds to the position where brackets were bonded $^{[7]}$. The outer prismless enamel layer might prevent infiltration of the phosphoric acid and allow shallow etching, ${ }^{[5,6]}$ resulting in insufficient penetration of the adhesive into the enamel surface. This deficient adhesive resin penetration might cause lower bond strengths in deciduous teeth than in permanent teeth ${ }^{[5]}$. Brackets bonded to deciduous and permanent teeth treated by the conventional acid etchant showed higher significant difference in shear bond strength, than those treated by the sulphated polyacrylic solution. This may indicate that, the difference in teeth composition and histology is more significant in the effect of phosphoric than sulphated polyacrylic acid.

Enamel surface pattern caused by etching with phosphoric acid in permanent and deciduous teeth can be explained by dissolving minerals in enamel. Etchants remove the outer 10 micrometers on the enamel surface 
and make a porous layer 5-50 micrometers deep. This roughens the enamel microscopically and results in a greater surface area on which to bond. Furthermore, the explanation for these different patterns between the two groups is most commonly attributed to different crystal orientation in the enamel ${ }^{[29]}$.

As a possible alternative to the conventional acid etching, crystal bonding technique with the application of polyacrylic acid containing residual sulphate ions reacts with the enamel surface to produce ionized carboxyl groups. Strong ionic bonding between calcium ions at the enamel surface and the carboxyl groups provides crystal enucleation sites for the gypsum crystals. This in turn provides micromechanical retention for the bonding resin ${ }^{[19,20]}$.

The reason for choosing this concentration $(1 \mathrm{M})$ based on other previous studies which concluded that there was no difference between the effect of using $20 \%-40 \%$ concentrations of polyacrylic acid and that experimental solutions containing $0.5 \mathrm{M}$ sulphate ion showed the peak of degree of crystal coverage ${ }^{[14,22]}$. Interesting finding of this study revealed comparable equal shear bond values between deciduous teeth treated with phosphoric acid and permanent teeth with crystalline growth solution (Group II and III). On the other hand, there was a significant difference between shear bond strength values produced with sulphated polyacrylic acid in deciduous teeth and permanent (Group I and III). This can be explained by the fact that deciduous enamel, exhibits morphological and histological differences: It is less-mineralized than permanent enamel, and it was proved that overall mineral density is lower in the outermost layers, but with no significant differences close to the enamelo-dentinal junction $^{[29]}$. Also, the base of the brackets in this study was designed to fit the premolar enamel surface configuration. The 3-dimensional surface configuration of deciduous molars is more complex than that of the premolars, causing the adhesive thickness between the bracket base and the deciduous enamel surface to increase. This increase in the adhesive thickness due to the complex deciduous enamel surface configuration could be another factor responsible for lowering the bond strength in the deciduous teeth, suggesting the need for a special type of brackets for deciduous teeth ${ }^{[26]}$. 
SEM confirmed the formation of calcium sulphated crystals when applying sulphated polyacrylic acid (1M) in both the deciduous and the permanent teeth, being more abundant on permanent teeth, possibly due to their higher inorganic content.

In this study, the sulphated polyacrylic acid applied to the deciduous and the permanent teeth showed higher frequencies of ARI scores 0 and 1, that interpreted by more clean enamel surfaces with no or minimal residual composite than did the conventional acid-etching adhesive system. This may strengthen the clinical advantages of using crystal growth solutions with less adhesive residues on enamel, and clinically acceptable bond strength value in the same time. This is translated into minimum cleaning after debonding and no or minimal harm to enamel structure.

Thus, despite that phosphoric acid etching is still the most efficient, it can be concluded that crystalline growth technique can be used for bonding brackets to deciduous as well as permanent teeth from the experimental in-vitro point of view. Further clinical investigations to study the reliability of using crystal growth solutions before bracket bonding in-vivo are strongly recommended.

\section{REFERENCES}

[1] Bishara S. JCO interviews on growth and orthodontic treatment. J Clin Orthod. 1998; 32(6):361-7.

[2] Endo T, Mizutani Y, Ozoe R, Shimooka S . Effects of early treatment of maxillary incisor crowding by using two bands and four brackets (2x4) mechanotherapy. Pediatr Dent J. 2005; 14:87-94.

[3] McKeown H, Sandler J. The two by four appliance: a versatile appliance. Dent Update. 2001; 28(10): 496-500.

[4] Tal E, Kupietzky A. Orthodontic alignment of permanent incisors following previous trauma of a deciduous tooth. Pediatr Dent. 2000; 22(1):71-2.

[5] Ripa LW, Gwinnett AJ, Buonocore MG. The "prismless" outer layer of deciduous and permanent enamel. Arch Oral Biol. 1966; 11(1):41-8.

[6] Gwinnett AJ. Human prismless enamel and its influence on sealant penetration. Arch Oral Biol. 1973; 18(3):441-4. 
[7] Whittaker DK. Structural variations in the surface zone of human tooth enamel observed by scanning electron microscopy. Arch Oral Biol. 1982; 27(5):383-92.

[8] Gwinnett AJ, Matsui A. A study of enamel adhesives. The physical relationship between enamel and adhesive. Arch OralBiol. 1967; 12(12):1615-20.

[9] LeFevre MN, Manly RS. Moisture, inorganic and organic contents of enamel and dentin from carious teeth. JADA and D Cos. 1938; 25:233-242.

[10] Armstrong WD, Brekhus PJ. Chemical constitution of enamel and dentin 1. Principle components. J Biol Chem. 1937; 120:677-687.

[11] Bird MJ, French EL, Woodside MR, Morrison MI, Hodge HC. Chemical analyses of deciduous enamel and dentin. J Dent Res. 1940; 19:413-23.

[12] Retief D, Busscher H, de Boer P, Jongebloed WL, Arends J. A laboratory evaluation of three etching solutions. Dent Mater. 1986; 2(5):202-6.

[13] Hobson R, McCabe J, Hogg S. Bond strength of surface enamel for different tooth types. Dent Mater. 2001; 17(2):184-9.

[14] Devanna, R, Keluskar, K. Crystal growth vs. conventional acid etching: A comparative evaluation of etch patterns, penetration depths, and bond strengths. Indian J Dent Res. 2008; 19(4): 309-14.

[15] Powers J, Messersmith M . Enamel etching and bond strength. In: Brantley W. and Eliades T (eds). Orthodontic Materials: Scientific and Clinical Aspects. New York, NY Thieme, 2001: 107-112.

[16] Øgaard B, Bishara S, Duschner H. Enamel effects during bondingdebonding and treatment with fixed appliances. In: Graber T, Eliades T, Athanasiou A (eds). Risk Managementin Orthodontics: Experts Guide to Malpractice. Carol Stream, Ill: Quintessence Publishing Co, Inc; 2004:19-46.

[17] Vicente A, Bravo L. and Romero, M. Influence of a non rinse conditioner on the bond strength of brackets bonded with a resin adhesive system. Angle Orthod. 2005; 75(3):400-5 
[18] Knox J, Jones ML. Crystal bonding--an adhesive system with a future? Br J Orthod. 1995; 22(4):309-17.

[19] Jones S, Gledhill, J, Davies E. The crystal growth technique- a laboratory evaluation of bond strengths. Eur J Orthod. 1999; 21(1): 89-93.

[20] Maijer R, Smith D. Crystal growth on the outer enamel surface-an alternative to acid etching. Am J Orthod. 1986; 89(3):183-93.

[21] Smith D, Lux, J, Maijer R. Crystal bonding to enamel. J Dent Res. 1981. 231: 368 .

[22] Kim JH, Lee KS. Effect of concentration of polyacrylic acid and sulphate ion on the crystal growth-Atopographic study. Kor J Orthod. 1998, 28:1-15.

[23] Northrup R, Berzins D, Bradley T, Schuckit W. Shear bond strength comparison between two orthodontic adhesives and self-ligating and conventional brackets. Angle Orthod. 2007; 77(4): 701-6.

[24] Reynolds I. Letter: 'Composite filling materials as adhesives in orthodontics'. Br Dent J. 1975; 138(3):83.

[25] Goksu T, and Oguz Oztoprak M. Plant extract ankaferd blood stopper effect on bond strength. Angle Orthod. 2010; 80(3):570-74.

[26] Endo T, Ozoe R, Shinkai K, Shimomura J, Katoh Y, Shimooka S. Comparison of shear bond strengths of orthodontic brackets bonded to deciduous and permanent teeth. Am J Orthod Dentofacial Orthop. 2008; 134(2):198-202.

[27] Endo T, Ozoe R, Shinkai K, Shimomura J, Katoh Y, Shimooka S. Shear bond strength of brackets rebonded with a fluoride-releasing and -recharging adhesive system. Angle Orthod. 2009; 79(3):564-70.

[28] Sokucu O, Siso SH, Ozturk F, Nalcaci R. Shear bond strength of orthodontic brackets cured with different light sources under thermocycling. Eur J Dent. 2010; 4(3):257-262.

[29] Wilson P, Beynon A. Mineralization differences between human deciduous and permanent enamel measured by quantitative micro-radiography. Arch Oral Biol. 1989, 34(2):85-88. 